This item was submitted to Loughborough's Research Repository by the author.

Items in Figshare are protected by copyright, with all rights reserved, unless otherwise indicated.

\title{
Attitude control of magnetically actuated satellites with an uneven inertia distribution
}

PLEASE CITE THE PUBLISHED VERSION

http://dx.doi.org/10.1016/j.ast.2011.12.005

PUBLISHER

(C) Elsevier Masson SAS

VERSION

AM (Accepted Manuscript)

LICENCE

CC BY-NC-ND 4.0

\section{REPOSITORY RECORD}

Wood, Mark, and Wen-Hua Chen. 2012. "Attitude Control of Magnetically Actuated Satellites with an Uneven Inertia Distribution”. figshare. https://hdl.handle.net/2134/11140. 
This item was submitted to Loughborough's Institutional Repository (https://dspace.lboro.ac.uk/) by the author and is made available under the following Creative Commons Licence conditions.

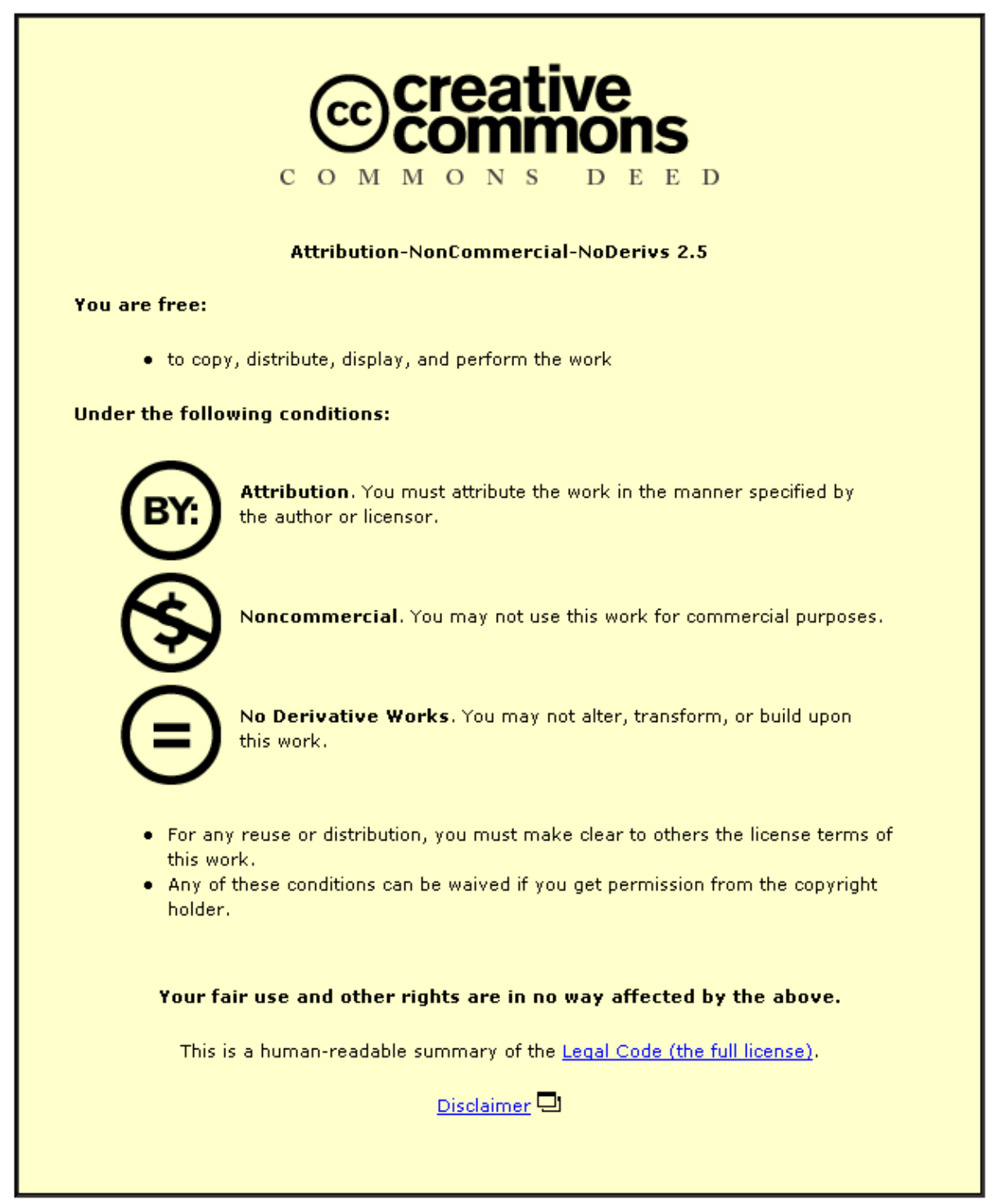

For the full text of this licence, please go to: http://creativecommons.org/licenses/by-nc-nd/2.5/ 


\title{
Attitude Control of Magnetically Actuated Satellites with
}

\section{An Uneven Inertia Distribution}

\author{
Mark Wood and Wen-Hua Chen \\ Department of Aeronautical and Automotive Engineering \\ Loughborough University \\ Loughborough. LE11 3TU, UK \\ Tel: +44 1509 227230; Fax: +44 1509227275 \\ w.chen@lboro.ac.uk
}

\begin{abstract}
This paper considers magnetic attitude control of a satellite with one axis of inertia significantly lower than that of the other two. With onboard resources often limited, this paper considers the development of an effective control strategy that remains easy to implement. Often used in this type of application, the classical 'torque-projection' approach is shown to be unsuitable for satellites with an uneven inertia distribution. To tackle the weaknesses in this approach a new 'weighted' PD approach is proposed, with the control torque determined through minimisation of a simple cost function. Through a similar philosophy, a feed-forward compensator is designed to supplement the feedback control and improve the disturbance rejection characteristics of the controller. Simulations carried out on a high fidelity model demonstrate the effectiveness of the proposed control law and the significant performance benefits offered over existing approaches.
\end{abstract}

Keywords: Magnetic Attitude Control; Satellite; PID Control; Feed-forward; Underactuated Control 


\section{INTRODUCTION}

The area of spacecraft magnetic attitude control is one that has attracted much recent attention in research literature. Use of magnetic dipoles to control the attitude of a spacecraft offers a lightweight, smooth, and cost-effective method of control. Although this is the case, the torque generated through use of magnetic dipoles is constrained to lie in the plane orthogonal to the local magnetic field vector, with one axis being instantaneously under-actuated. If the satellite is on an inclined orbit, suitable variation of the magnetic field allows controllability in the long term, but presents a significant challenge from a control perspective.

Within the research literature a whole array of differing control strategies are proposed to deal with the magnetic attitude control problem. One of the simplest and most common control strategies is the 'torque projection' PD controller. An ideal control torque is calculated using a PD controller, with the assumption that full controllability is available. This ideal torque cannot be directly applied and as a design choice is projected onto the plane orthogonal to the Earth's magnetic field where the resulting torque can be realized. Firstly proposed in reference [1] and more recently in reference [2], the simplicity and ease of implementation of this controller has seen widespread industrial application.

Although easy to implement, the use of PD control cannot always provide the required performance and as a result several authors investigate more advanced control strategies. The pseudo-periodic nature of the Earth's magnetic field has led to a number of studies into the use of optimal periodic control theory. Once of the earliest studies into infinite 
horizon periodic control for the satellite attitude control problem is presented in reference [3]. The optimal controller gains are obtained offline through solution of the periodic Riccati equation and then stored in onboard memory for implementation. Reference [4] applies a similar optimal periodic control approach to the problem while providing an interesting extension to include optimal disturbance rejection. This approach proposes online solution to the Riccati equation rather than onboard storage of controller gains. Reference [5] presents the most significant work in this area, as the issue of onboard computation and storage requirements is avoided. Under certain assumptions the solution of the periodic Riccati equation is shown to tend to a constant value. This assumption allows the periodic gain to be calculated online with minimal computation. This approach is only slightly limited by the fact that it only remains valid for satellites with an openloop stable configuration.

The interest in infinite horizon control laws has naturally led to investigations into finite horizon applications. Model predictive control (MPC) has been identified as a suitable candidate for dealing with the magnetic attitude problem in several recent papers. Reference [6] presents the first notable contribution in this area, with the constraint handling of MPC exploited in a novel way. The control problem is formed in a time invariant manner with the time varying nature of the actuation introduced through an appropriate set of equality constraints. This helps to reduce the computational burden of the strategy as calculations of prediction matrices can be carried out offline. Reference [7] applies a similar control approach, while extending the controller to consider disturbance rejection. The disturbance rejection properties of the MPC strategy are noticeably 
improved without increasing the complexity of the controller. Most recently reference [8] considers a different approach to the problem to address the problem of stability. A stability enforced predictive controller is shown to guarantee asymptotic stability of the closed-loop even for satellite with unstable open-loop pitch dynamics.

Although more advanced control techniques such as optimal control and MPC undoubtedly provide improved performance over classic PD approaches, many magnetically actuated satellites do not have the onboard resources to implement the complex algorithms often associated with these designs. Magnetic actuation is often used on small, low-cost satellites where the onboard hardware is extremely limited. For many of these cases the pointing accuracy is also relaxed making the torque projection approach very attractive.

Although the torque projection approach is suitable for satellites for which the principal axes of inertia are of the same order of magnitude, the success of the control strategy is significantly degraded if one axis of inertia is significantly lower than that of the other two. Reference [9] reports difficulties when applying the torque projection approach to such a configuration and presents a modified, albeit fairly ad-hoc approach to adjust the pitch gain as a function of the local magnetic field vector. The act of projecting the torque onto the Earth's magnetic field orthogonal places a low priority on the low inertia axis (a point first identified in this paper), with performance about this axis significantly degraded. 
The aim of this paper is to provide a systematic approach to the design of magnetic attitude control systems of satellites with an uneven inertia distribution. This is done while maintaining simplicity of the control law, presenting an effective and feasible control approach. This paper is structured as follows. Section 2 introduces the benchmark satellite and dynamic equations. With the attitude regulation problem being considered, a linear model is sufficient to fully describe the attitude dynamics. Section 3 identifies the weaknesses in the torque projection PD approach when applied to a satellite with an uneven inertia distribution. A new control approach is then proposed to address these weaknesses. Section 4 augments the newly proposed feedback control with a feed-forward compensator. In a similar way the feed-forward control is carefully designed to correctly tackle the uneven inertia distribution. Section 5 presents a simulation study to demonstrate the effectiveness of the proposed control approach using a high fidelity simulation model.

\section{SPACECRAFT DYNAMICS UNDER MAGNETIC CONTROL}

Set for launch in 2009, the GOCE satellite is part of the European Space Agency's (ESA) living planet program. The main aim of the satellite is to measure the Earth's gravity field gradient and hence the control aim is to cancel all non-gravitational angular accelerations acting upon GOCE. The selected orbit is a sun-synchronous, near-polar orbit of $96.5^{\circ}$ inclination, at an altitude of $250 \mathrm{~km}$, with an orbital period of approximately $5400 \mathrm{~s}$. The inertia distribution is summarized in Table 1, leading to an unstable pitch and neutrally stable lateral configuration. For attitude regulation GOCE is equipped with three mutually perpendicular magnetic dipole rods each with $400 \mathrm{Am}^{2}$ saturation limit. The GOCE satellite has fairly relaxed pointing requirements of $8^{\circ}$ about the roll and yaw axes and $3^{\circ}$ 
about the pitch axis. The interested reader may consult reference [9] for further information on the GOCE mission.

Although the true spacecraft dynamics are fully described by a series of non-linear differential equations, under certain assumptions these equations can be linearized with minimal loss of accuracy. If linearization is carried out about the equilibrium nadirpointing attitude, assuming a circular orbit, small Euler angles and deviation of body rates from nominal values, the following linearized model can be produced [5]. Once a satellite has acquired an Earth pointing attitude on orbit (i.e. once the initial high pointing angle and angular rates have been removed through non-linear control), the satellite dynamics are approximated well by such a linear model.

$$
\dot{x}(t)=\mathbf{A}_{\mathrm{c}} x(t)+\mathbf{B}_{\mathrm{c}} T(t)
$$

where

$$
\begin{aligned}
& \mathbf{A}_{\mathbf{c}}=\left[\begin{array}{cccccc}
0 & 0 & 0 & 1 & 0 & 0 \\
0 & 0 & 0 & 0 & 1 & 0 \\
0 & 0 & 0 & 0 & 0 & 1 \\
-4 \omega_{0}{ }^{2} \sigma_{1} & 0 & 0 & 0 & 0 & \omega_{0}\left(1-\sigma_{1}\right) \\
0 & 3 \omega_{0}{ }^{2} \sigma_{2} & 0 & 0 & 0 & 0 \\
0 & 0 & \omega_{0}{ }^{2} \sigma_{3} & -\omega_{0}\left(1+\sigma_{3}\right) & 0 & 0
\end{array}\right], \mathbf{B}_{\mathbf{c}}=\left[\begin{array}{c}
\mathbf{0}_{3,3} \\
\mathbf{J}^{-1}
\end{array}\right] \\
& \boldsymbol{x}(\boldsymbol{t})
\end{aligned}
$$

$\phi, \theta, \psi$ are the spacecraft pointing angles about roll, pitch and yaw axes respectively, $\omega_{x}, \omega_{y}, \omega_{z}$ are the spacecraft angular rates about roll, pitch and yaw axes respectively, 
$\omega_{0}$ is the orbital rate, $\mathbf{J}$ is the inertia matrix, $\boldsymbol{T}$ is the control torque (with components $\left.T_{x}, T_{y}, T_{z}\right)$, and $\sigma_{i}=\left(J_{j}-J_{k}\right) / J_{i}$ for the index sets $(1,2,3),(2,3,1)$ and $(3,1,2)$.

Note that the co-ordinate system used throughout this paper defines the spacecraft orientation relative to a local-level co-ordinate system. The local-level system has the $+z$ axis pointing towards the nadir, the $y$ axis perpendicular to the orbital plane (defined by position and velocity vector), and the $x$ axis defined by the right hand rule.

When considering magnetically controlled spacecraft, torque-rods generate control torques through interaction with the Earth's own magnetic field. This torque is perpendicular to the Earth's magnetic field vector and is described below in equation (2).

$$
\boldsymbol{T}=\boldsymbol{M} \times \boldsymbol{B}_{m a g}
$$

where $\boldsymbol{M}$ is the vector of magnetic dipole moments (with components $M_{x}, M_{y}, M_{z}$ ) and $\boldsymbol{B}_{\text {mag }}$ is the Earth's magnetic field vector (with components $B_{\text {mag }_{x}}, B_{\text {mag }_{y}}, B_{\text {mag }_{z}}$ ). 


\section{PD ATTITUDE CONTROL}

\subsection{Torque Projection PD Control}

A simple but effective technique of implementing magnetic control is through the socalled "torque-projection" approach. Originally proposed in reference [1] a PD controller is used to calculate a required control torque through equation (3)

$$
\boldsymbol{T}_{\text {ideal }}=-\mathbf{K} \boldsymbol{x}
$$

where $\mathbf{K}$ is a $3 \times 6$ gain matrix.

Due to the magnetic field constraints the torque in equation (3) cannot be implemented directly and as a design choice this torque is projected onto the plane orthogonal to the Earth's magnetic field. The resulting magnetic dipole and torque vectors are described below in equations (4) and (5).

$$
\begin{aligned}
& M=\frac{B_{\text {mag }} \times T_{\text {ideal }}}{\left|B_{\text {mag }}\right|^{2}} \\
& T_{\text {true }}=\left(I_{3}-\frac{B_{\text {mag }} B_{\text {mag }}^{T}}{B_{\text {mag }}^{T} B_{\text {mag }}}\right) T_{\text {ideal }}
\end{aligned}
$$

where $\mathbf{I}_{\mathbf{3}}$ is the $3 \times 3$ identity matrix.

Although usually considered as a geometric approximation, derivation of the control signal in this manner is in fact an implementation of a simple optimisation problem. 
Consider minimising the following quadratic performance index

$$
\min \left\|\boldsymbol{T}_{\text {ideal }}-\boldsymbol{T}_{\text {true }}\right\|^{2}
$$

subject to the constraint

$$
\boldsymbol{B}_{\text {mag }} \cdot \boldsymbol{T}_{\text {true }}=0
$$

The solution of equation (6) subject to the constraint in equation (7) leads to the same torque as calculated in equation (5) and effectively minimises the Euclidean norm of the error between the ideal and true control torques. For many satellite configurations this approach is sensible and leads to acceptable results. For satellites with an uneven inertia distribution the success of this approach diminishes. The reasons for the degradation of this approach are best illustrated with a numerical example.

Consider the design of a torque projection PD controller for the GOCE satellite. The ideal feedback gain is chosen through a pole placement technique, with the ideal closed-loop poles placed with damping ratio of 0.7 and natural frequencies of $3.5 \omega_{0}, 0.5 \omega_{0}$ and $0.25 \omega_{0}$. The resulting feedback gain matrix is shown in equation (8).

$$
\boldsymbol{K}=\left[\begin{array}{cccccc}
-1.7 e-4 & 0 & -2.7 e-9 & 0.094 & 0 & 0.17 \\
0 & 5.5 e-2 & 0 & 0 & 15.6 & 0 \\
-4.8 e-8 & 0 & -3 e-3 & -0.68 & 0 & 1.6
\end{array}\right]
$$


For illustrative purposes assume the Earth's magnetic field can be represented using the dipole model shown below.

$$
\boldsymbol{B}_{\text {mag }}=\frac{\mu_{f}}{a^{3}}\left[\begin{array}{c}
\cos \omega_{0} t \sin i_{m} \\
-\cos i_{m} \\
2 \sin \omega_{0} t \sin i_{m}
\end{array}\right]
$$

where $\mu_{f}=7.9 \times 10^{15} \mathrm{~Wb} . \mathrm{m}$ is the dipole field strength, $a$ is the orbit height and $i_{m}$ is the orbital inclination.

For simplicity assume an orbital inclination of $i_{m}=\pi / 2$ and time of $t=2 \pi / 3 \omega_{0}$. The resulting magnetic field vector is calculated as $\boldsymbol{B}_{\text {mag }}=\left[\begin{array}{llll}-1.36 e-5 & 0 & 4.70 e-5\end{array}\right]^{T}$. Also assume a typical state vector of $x=\frac{\pi}{180}\left[\begin{array}{llllll}1 & 0.01 & 1 & 1 e-3 & 1 e-4 & 1 e-3\end{array}\right]^{T}$. Applying the control law in equations (3) and (5) the commanded and implemented torque are summarised in Table 2.

The first point to note from Table 2 is that the pitch torque is fully implemented. As the pitch axis is always controllable through one of the lateral dipole moments, this demonstrates the torque projection approach to be suitable for regulation of the pitch axis. Focussing now on the roll and yaw performance it is clear that the commanded and implemented torque are now quite different. This is not a surprising result as the restrictions due to the magnetic field will obviously restrict the authority of the controller. The main concern comes when analysing the numerical values of these torques and the resulting angular accelerations. 
A torque of $36 \mu \mathrm{Nm}$ is commanded about the yaw axis which, when normalising with respect to the yaw inertia gives a commanded acceleration of $1.3 \times 10^{-8} \mathrm{rad} / \mathrm{s}^{2}$. Once the torque projection has been carried out the implemented torque is reduced to just $2 \mu \mathrm{Nm}$ with a corresponding angular acceleration of $7.5 \times 10^{-10} \mathrm{rad} / \mathrm{s}^{2}$. For the roll axis a torque of $-1.5 \mu \mathrm{Nm}$ is commanded which, when normalised with respect to the roll inertia gives a commanded acceleration of $-1.0 \times 10^{-8} \mathrm{rad} / \mathrm{s}^{2}$. Note that this acceleration is similar in magnitude to that commanded about the yaw axis even though the commanded control torque is much smaller. Once the torque projection has been carried out the torque implemented is $+8 \mu \mathrm{Nm}$. This corresponds to an angular acceleration of $5.2 \times 10^{-8} \mathrm{rad} / \mathrm{s}^{2}$, a value five times larger and in the opposite direction to that commanded. Although the controller is minimising the overall torque error, no consideration is placed on the fact that torque errors in the roll axis induce much larger acceleration errors due to the lower satellite inertia. This numerical problem leads to poor performance about the low inertia axis and needs to be addressed when considering control of satellites with uneven inertia distribution.

\subsection{Weighted PD Control}

The numerical example in the previous section shows that when minimising the Euclidean norm of the error between the true and ideal control torques, the low inertia axis is given low priority within this minimisation process. Although the overall torque error is minimised the effect about the roll axis can be significant due to the lower inertia about this axis. To tackle this weakness in the torque projection approach a modification to the controller is proposed. 


\subsubsection{Derivation of Weighted PD Controller}

Instead of minimising the Euclidean norm of the torque error, consider minimisation of the following weighted performance index

$$
\min \frac{1}{2}\left(\boldsymbol{T}_{\text {ideal }}-\boldsymbol{T}_{\text {true }}\right)^{T} \mathbf{Q}\left(\boldsymbol{T}_{\text {ideal }}-\boldsymbol{T}_{\text {true }}\right)
$$

subject to the constraint

$$
\boldsymbol{B}_{\text {mag }} \cdot \boldsymbol{T}_{\text {true }}=0
$$

where $\mathbf{Q}>0$ is a diagonal weighting matrix

As the term $\frac{1}{2} \boldsymbol{T}_{\text {ideal }}^{T} \mathbf{Q} \boldsymbol{T}_{\text {ideal }}$ is a constant, the optimisation problem in (10) and (11) can be simplified to the following form.

$$
\min \left(-\boldsymbol{T}_{\text {ideal }}^{T} \mathbf{Q} \boldsymbol{T}_{\text {true }}+\frac{1}{2} \boldsymbol{T}_{\text {true }}^{T} \mathbf{Q} \boldsymbol{T}_{\text {true }}\right)
$$

subject to the constraint

$$
\boldsymbol{B}_{\text {mag }} \cdot \boldsymbol{T}_{\text {true }}=0
$$

As equations (12) and (13) are now in the form of a general quadratic programming problem subject to an equality constraint, a closed-form solution can be derived through 
use of Lagrange multipliers (see reference [10]). The necessary Lagrange conditions for this problem are described in equations (14) and (15).

$$
\begin{aligned}
& \mathbf{Q} \boldsymbol{T}_{\text {true }}+\boldsymbol{B}_{\text {mag }} \lambda-\mathbf{Q} \boldsymbol{T}_{\text {ideal }}=0 \\
& \boldsymbol{B}_{\text {mag }}^{T} \boldsymbol{T}_{\text {true }}=0
\end{aligned}
$$

where $\lambda$ is the Lagrange multiplier.

From equation (14)

$$
\boldsymbol{T}_{\text {true }}=-\mathbf{Q}^{-1} \boldsymbol{B}_{\text {mag }} \lambda+\boldsymbol{T}_{\text {ideal }}
$$

Substituting equation (16) into equation (15) leads to

$$
-B_{m a g}^{T} Q^{-1} B_{m a g} \lambda+B_{m a g}^{T} T_{i d e a l}=0
$$

Solving for the Lagrange multiplier gives

$$
\lambda=\left(\boldsymbol{B}_{\text {mag }}^{T} \mathbf{Q}^{-1} \boldsymbol{B}_{\text {mag }}\right)^{-1} \boldsymbol{B}_{\text {mag }}^{T} \boldsymbol{T}_{\text {ideal }}
$$

Finally substitution of equation (18) into equation (16) yields the final solution

$$
\boldsymbol{T}_{\text {true }}=\boldsymbol{T}_{\text {ideal }}-\mathbf{Q}^{-\mathbf{1}} \boldsymbol{B}_{\text {mag }}\left(B_{\text {mag }}^{T} \mathbf{Q}^{-1} B_{\text {mag }}\right)^{-1} B_{\text {mag }}^{T} \boldsymbol{T}_{\text {ideal }}
$$


For many optimisation problems the matrix inverse in equation (19) makes the problem not numerically attractive as the matrix $\left(\boldsymbol{B}_{\text {mag }}^{T} \mathbf{Q}^{-\mathbf{1}} \boldsymbol{B}_{\text {mag }}\right)$ is often ill-conditioned. For this specific optimisation problem this issue is removed as the aforementioned matrix is a scalar value such that

$$
\left(\boldsymbol{B}_{m a g}^{T} \mathbf{Q}^{-1} \boldsymbol{B}_{m a g}\right)^{-1}=\frac{B_{\text {mag }_{x}}^{2}}{q_{1}}+\frac{B_{m a g_{y}}^{2}}{q_{2}}+\frac{B_{m a g_{z}}^{2}}{q_{3}}
$$

As the Earth's magnetic field vector will never equal zero in all directions $\left(\boldsymbol{B}_{m a g}^{T} \mathbf{Q}^{-1} \boldsymbol{B}_{m a g}\right)^{-1}>0$ if $q_{1}, q_{2}, q_{3}>0$. Equation (19) can therefore be written in the more compact form

$$
T_{\text {true }}=\left(I_{3}-\frac{\mathbf{Q}^{-1} B_{m a g} B_{m a g}^{T}}{B_{m a g}^{T} \mathbf{Q}^{-1} B_{m a g}}\right) T_{i d e a l}
$$

The dipole moment required to implement this torque is determined by taking the cross product of the magnetic field vector and the Earth's magnetic field vector according to equation (22).

$$
M=\frac{B_{m a g} \times T_{t r u e}}{\left|B_{m a g}\right|^{2}}
$$


The tuning process itself can be simplified by considering the case of a satellite with one axis of inertia considerably lower than that of the other two. For the case of GOCE it is the weighting of the roll axis relative to the pitch and yaw axes that is of importance and the Q matrix can therefore be specified as

$$
\mathbf{Q}=\left[\begin{array}{lll}
q & 0 & 0 \\
0 & 1 & 0 \\
0 & 0 & 1
\end{array}\right]
$$

where $q>0$ is the roll weighting factor.

\subsubsection{Numerical Example}

To illustrate the benefits of the newly proposed weighted PD control, the numerical example from the previous section is revisited. The same magnetic field, state vector and ideal feedback gain are chosen, but the control signal is now implemented using equation (21). The roll weighting factor is chosen as $q=8$ and the results are shown in Table 3 .

This control approach does not miraculously allow control of both axes, but does provide a more intelligent method of implementing the control signal. Consider once again the torque about the yaw and roll axes; the implemented torque about the yaw axis is now slightly degraded from the torque projection approach, but this degradation is relatively insignificant when considering the large inertia of this axis. The control input applied about the roll axis is now much more sensible. The commanded value of $-1.64 \mu \mathrm{Nm}$ cannot be achieved due to the magnetic field constraints however, due to the increased weighting within the optimisation process, the controller avoids applying a large acceleration in the 
opposite direction to that commanded. At this particular orbit location and state vector, implementation of the desired control signal is not possible regardless of how the torque is selected. Use of the weighted PD approach avoids inducing large unwanted accelerations about the low inertia axis and is done at relatively small penalty to the yaw axis.

The numerical example in Table 3 illustrates the motivation for the weighted PD approach. The true benefits of adopting a weighted PD controller are best demonstrated through a simulation study. The GOCE satellite is initialised at $1^{\circ}$ pointing about each satellite axis and is considered under both torque projection PD control and the newly proposed weighted PD control. The ideal feedback gain is as defined in equation (8) and the roll weighting factor is chosen to be 8 .

The results shown in Figure 1 support the numerical example detailed in Table 3. When both the roll and yaw errors are large, the torque projection controller places more emphasis on removal of the yaw error. This is at significant detriment to the roll axis, which at worst reaches $9^{\circ}$ pointing error from an initial value of just $1^{\circ}$. As the yaw error reduces, the controller is able to restore the roll attitude, but this is clearly unacceptable performance. When the newly proposed weighted PD controller is used, the performance about the two axes is more comparable. The yaw performance is degraded (as would be expected), but this is far outweighed by the significant improvement in performance about the roll axis. 


\subsubsection{Roll Weighting Factor}

The introduction of the weighted PD controller provides an additional tuning parameter to the control engineer. Selection of an appropriate weighting value is dependent on several factors.

- The ideal feedback gain: Selection of the ideal feedback gain determines the magnitude of the commanded control torques. As already discussed, it is the difference in the magnitude of the commanded torques that causes the numerical problems that bias the controller in favour of the higher inertia axes. The manner in which this feedback gain is chosen affects the control torques, and hence the weighting required to obtain acceptable performance about the low inertia axis.

- The level of performance required about the low inertia axis: If the pointing accuracy about the low inertia axis is of low importance, a low $q$ value may be suitable to place more emphasis on performance about the other axes. If more accurate pointing is required about the low inertia axis the $q$ value may need to be much higher for optimal performance.

- External disturbances: The level of external disturbance is very much dependent on the satellite configuration and orbit height. As well as providing suitable nominal response the feedback control must provide sufficient disturbance rejection. If too much weighting is placed on a given axis this will reduce the disturbance rejection capabilities of the other axes and can degrade performance of the overall system. 
The selection of the roll weighting factor is application specific and should be determined through a short trial and error process. The effect of varying the weighting is demonstrated in Figure 2 .The satellite is initially at pointing angles of $1^{\circ}$ about each axis and is controlled using a weighted PD strategy with varying $q$ value.

Selection of a low weighting factor $(q=1)$ places more significance on the higher inertia axes within the optimisation. As already discussed this can lead to poor performance about the low inertia axis. Increasing the weighting factor $(q=8)$ significantly improves performance about the low inertia axis at a cost of slight degradation to the yaw axis. Increasing the roll weighting further $(q=50)$ places further emphasis on the low inertia axis and degrades the performance about the yaw axis.

The most important point here is that each response shown in Figure 2 is achieved at the same ideal feedback gain. Once the ideal feedback gain has been selected, addition of the roll feedback weighting introduces a very intuitive tuning parameter that can be used to improve performance about the low inertia axis. 


\section{FEEDFORWARD DISTURBANCE COMPENSATION}

For satellites operating on a low Earth orbit, disturbances due to the external environment have a significant effect on the time response of the attitude dynamics. The feedback control in the previous section can be augmented with a feed-forward element to improve the disturbance rejection properties of the overall attitude controller. Clearly these environmental torques cannot be measured directly and must be estimated using a Kalman filter. As GOCE is also without rate gyros this state estimator must also provide angular rate information.

\subsection{Kalman Filter}

To provide estimates of the external disturbances a mathematical model of these torques is required. A precise model of these torques is fully obtained using a high order Fourier decomposition, however from the perspective of an observer design this is obviously restrictive. Fortunately analysis of the external environment shows that the most significant harmonics occur at frequencies $0, \omega_{0}$ and $2 \omega_{0}{ }^{[11]}$. Furthermore, reference [7] notes that suitable estimates of the external disturbances can be achieved by assuming the disturbances remain constant with time. This assumption is therefore used here to maintain simplicity of the state observer design and to minimise onboard computation of the state estimates. Consider the discrete-time constant disturbance model

$$
\boldsymbol{d}(k+1)=\boldsymbol{d}(k)+\boldsymbol{v}(k)
$$

where $\boldsymbol{d}$ is the external disturbance and $\boldsymbol{v}$ is white noise. 
The continuous-time dynamics in equation (1) can also be discretised to provide the following model

$$
\boldsymbol{x}(k+1)=\mathbf{A} \boldsymbol{x}(k)+\mathbf{B} \boldsymbol{T}(k)+\boldsymbol{d}(k)
$$

where,

$$
\mathbf{A}=\exp \left(\mathbf{A}_{\mathbf{c}} T_{s}\right), \mathbf{B}=\left[\int_{0}^{T_{s}} \exp \left(\mathbf{A}_{\mathbf{c}} \eta\right) d \eta\right] \mathbf{B}_{\mathbf{c}}, T_{s} \text { is the estimator sampling time }
$$

The relationships in equations (24) and (25) can be formed into an augmented plant model to provide the basis of the state observer.

$$
\begin{aligned}
& \boldsymbol{x}_{e}(k+1)=\mathbf{A}_{e} \boldsymbol{x}_{e}(k)+\mathbf{B}_{e} \boldsymbol{u}(k)+\left[\begin{array}{c}
\mathbf{0}_{\mathbf{6}, \mathbf{3}} \\
\mathbf{I}_{\mathbf{3}}
\end{array}\right] \boldsymbol{v}(k) \\
& \boldsymbol{y}_{\boldsymbol{e}}(k+1)=\boldsymbol{C}_{\boldsymbol{e}} \boldsymbol{x}_{e}(k)+\boldsymbol{D}_{e} \boldsymbol{u}(k)+\boldsymbol{w}(k)
\end{aligned}
$$

where

$$
\begin{aligned}
\mathbf{A}_{e} & =\left[\begin{array}{cc}
\mathbf{A} & \mathbf{B} \\
\mathbf{0}_{3,6} & \mathbf{I}_{\mathbf{3}}
\end{array}\right], \quad \mathbf{B}_{\mathbf{e}}=\left[\begin{array}{c}
\mathbf{B} \\
\mathbf{0}_{3,3}
\end{array}\right], \quad \boldsymbol{x}_{e}(k)=\left[\begin{array}{c}
\boldsymbol{x}(k) \\
\boldsymbol{d}(k)
\end{array}\right] \\
C_{e} & =\left[\begin{array}{cc}
\mathbf{A} & \mathbf{B} \\
\mathbf{0}_{\mathbf{3}, \mathbf{6}} & \mathbf{I}_{\mathbf{3}}
\end{array}\right] \boldsymbol{D}_{\boldsymbol{e}}=\left[\begin{array}{l}
\mathbf{0}_{3,3} \\
\mathbf{J}^{-\mathbf{1}}
\end{array}\right]
\end{aligned}
$$

An estimate of the augmented state can then be provided through equation (28)

$$
\hat{\boldsymbol{x}}_{e}(k+1)=\mathbf{A}_{e} \hat{\boldsymbol{x}}_{e}(k)+\mathbf{B}_{e} \boldsymbol{u}(k)+\mathbf{L}_{\mathbf{e}}\left[\boldsymbol{y}(k)-\left(\mathbf{C}_{\mathbf{e}} \hat{\boldsymbol{x}}_{e}(k)+\mathbf{D}_{\mathbf{e}} \boldsymbol{u}(k)\right)\right]
$$


where $\mathbf{L}_{\mathbf{e}}$ is the Kalman filter gain matrix and $\boldsymbol{y}(k)$ is the measured position and acceleration taken directly from star sensor and accelerometer measurements.

\subsection{Weighted Feed Forward Compensation}

The methodology in Section 3 can now be naturally extended when considering the design of an appropriate feed-forward strategy. The ideal feed-forward control will take the estimate of the external disturbance provided by the Kalman filter and implement an equal and opposite torque to cancel this unwanted disturbing moment. As magnetic torquers are used as actuators this is not possible and the effect of the external disturbances must be minimised as much as possible.

The ideal control torque to apply is described in equation (29).

$$
\boldsymbol{T}_{\boldsymbol{F F}_{\text {ideal }}}(k)=-\left[\begin{array}{ll}
\mathbf{0}_{\mathbf{3 , 6}} & \mathbf{I}_{3}
\end{array}\right] \hat{\boldsymbol{x}}_{\boldsymbol{e}}(k)
$$

Based on current design methods, an intuitive approach is to project this ideal torque onto the controllable plane in a manner analogous to the torque projection PD controller. Once again for satellites with a similar inertia about each axis this is a suitable approach, but when considering satellites with an uneven inertia distribution this approach can actually degrade performance. As a result the feed-forward input is determined though minimisation of the following performance index.

$$
\min \frac{1}{2}\left(\boldsymbol{T}_{F F_{\text {iddal }}}-\boldsymbol{T}_{F F_{\text {true }}}\right)^{T} \mathbf{Q}_{F F}\left(\boldsymbol{T}_{F F_{\text {ideal }}}-\boldsymbol{T}_{F F_{\text {true }}}\right)
$$


As the aim of the feed-forward controller is to minimise the effects of the external disturbances, more analytical guidance can be given to choose the $\mathbf{Q}_{F F}$ matrix. As a design choice the effect of the external disturbance is quantified in terms of the angular accelerations imparted on the spacecraft. After the disturbance compensation has been applied the acceleration torque seen by the spacecraft is

$$
a=\mathbf{J}^{-1}\left(T_{F F_{\text {ideal }}}-T_{F F_{\text {true }}}\right)
$$

The cost function to be minimised is therefore

$$
\min \frac{1}{2} a^{T} a
$$

If $J_{y} \approx J_{z}$ this is equivalent to choosing the $\mathbf{Q}_{F F}$ matrix as follows

$$
\mathbf{Q}_{\mathbf{F F}}=\left[\begin{array}{ccc}
q_{f f} & 0 & 0 \\
0 & 1 & 0 \\
0 & 0 & 1
\end{array}\right]
$$

where $q_{f f}=\left(J_{z} / J_{x}\right)^{2}$

This approach to selection of the weighting matrix cannot be successfully applied to the feedback portion of the control due to the differing aims of the two control components. As the aim of the feedback control is to provide suitable closed-loop dynamics, minimisation of acceleration errors between the true and ideal case is not a meaningful 
design approach (minimising the acceleration error does not guarantee any particular expected closed-loop behaviour). For the feed-forward case, minimisation of accelerations is a sensible measure and hence the proposed method of selecting the $\mathbf{Q}_{F F}$ matrix.

\subsubsection{Numerical Example}

Once again the motivation for the weighted feed-forward compensator can be illustrated with a simple numerical example. Consider the satellite at the same orbital location used in the previous examples, subject to a disturbance of $1 \times 10^{-5} \mathrm{Nm}$ about the pitch and yaw axes and $1 \times 10^{-6} \mathrm{Nm}$ about the roll axis.

Table 4 illustrates the problems associated when the feed-forward compensation is not correctly designed. In attempting to minimise the disturbance torque about the yaw axis the torque-projection control increases the disturbance acting on the low inertia axis. This imparts large accelerations about the low inertia axis and can actually degrade the performance of the controller. The weighted feed-forward controller penalises the accelerations on each axis rather than the control torque, so ensures the low inertia axis is more appropriately dealt with. The disturbance about this axis is almost completely removed, while still removing the pitch disturbance and part of the yaw disturbance.

The numerical example in Table 4 can also be reinforced with a simple simulation study. The GOCE satellite is initially at $1^{\circ}$ pointing about each axis and is regulated using the weighted PD feedback control proposed in the previous section. The satellite is subject to a constant disturbance of $1 \times 10^{-5} \mathrm{Nm}$ about the pitch and yaw axes and $1 \times 10^{-6} \mathrm{Nm}$ about 
the roll axis. In order to improve the disturbance rejection properties the feedback control is augmented with a torque-projection feed-forward controller and the newly proposed weighted feed-forward controller.

If the state estimator in equation (28) is used the final control law combining the weighted feedback and feed-forward schemes simplifies to the following.

$$
\boldsymbol{T}=-\overline{\mathbf{K}} \hat{\boldsymbol{x}}_{e}
$$

where

$$
\overline{\mathbf{K}}=\left(\left(\mathbf{I}_{3}-\frac{\mathbf{Q}^{-1} \boldsymbol{B}_{m a g} \boldsymbol{B}_{m a g}^{T}}{\boldsymbol{B}_{m a g}^{T} \mathbf{Q}^{-1} \boldsymbol{B}_{m a g}}\right) \mathbf{K}\left[\mathbf{I}_{6} \quad \mathbf{0}_{6,3}\right]+\left(\mathbf{I}_{3}-\frac{\mathbf{Q}_{\mathrm{FF}}^{-1} \boldsymbol{B}_{m a g} \boldsymbol{B}_{m a g}^{T}}{\boldsymbol{B}_{m a g}^{T} \mathbf{Q}_{\mathrm{FF}}^{-1} \boldsymbol{B}_{m a g}}\right)\left[\begin{array}{ll}
\mathbf{0}_{3,6} & \mathbf{I}_{3}
\end{array}\right]\right)
$$

Figure 3 shows the response under the control law in equation (34). With feedback only control the external disturbance causes a large steady state error about the roll axis, while performance about the yaw axis is much better due to its higher inertia. The addition of the torque-projection feed-forward controller improves performance about the yaw axis however this is at the expense of the low inertia roll axis. Use of the weighted feedforward control allows for slight improvement about the yaw axis but significantly improves the roll performance. The steady state error is removed and the nadir pointing attitude is regulated more successfully. When adopting torque projection feed-forward control, the (already well regulated) yaw axis is given high priority. The addition of the weighted feed-forward control penalises the accelerations due to the external disturbances and thus allows fairer consideration of the low inertia roll axis. 


\section{SIMULATION}

\subsection{Simulation Environment}

To fully validate the proposed control approach, the GOCE satellite is simulated using a high fidelity simulation model. Although the linear model in equation (1) has been used for controller design, a full non-linear description of the satellites dynamics is used for numerical simulation. The Earth's magnetic field is modelled using an $8^{\text {th }}$ order IGRF model to achieve suitably high accuracy.

Due to its low Earth orbit GOCE is subject to aerodynamic drag from the upper atmosphere. This causes a perturbing torque within the attitude dynamics as the drag line is offset from the spacecraft centre of mass. This also leads to the need for an ion thruster assembly (ITA) to counteract this drag force and maintain orbital speed. This in turn further disturbs the attitude dynamics. Ideal measurements of acceleration and pointing angle are corrupted with sensor noise, while the lack of rate gyros is represented and any rate information used within the control law is obtained from the Kalman filter in equation (28).

\subsection{Simulation}

The effectiveness of the proposed control strategy can now be assessed on the high fidelity GOCE simulator. Firstly the weighted PD controller is implemented without the feedforward compensation. This allows a fair comparison to be made between the torque projection PD controller and the weighted PD controller while also demonstrating the contribution made by the feed-forward term once included. The roll weighting factor for 
the weighted PD approach is chosen as $q=8$ with the ideal feedback gain matrix for both approaches chosen as

$$
\boldsymbol{K}=\left[\begin{array}{cccccc}
1 e-5 & 0 & 0 & 0.2 & 0 & 0 \\
0 & 5.5 e-2 & 0 & 0 & 15.6 & 0 \\
0 & 0 & 1 e-5 & 0 & 0 & 1.6
\end{array}\right]
$$

The satellite is initialised with $1^{\circ}$ pointing about each axis. Figure 4 shows that, as demonstrated by earlier examples, the torque projection control places little emphasis on the low inertia roll axis and leads to large pointing errors. The significant external disturbances acting on the GOCE satellite highlight this point with the maximum roll error reaching up to $30^{\circ}$. Use of the weighted PD controller provides a more suitable approach to the control problem, with the pointing accuracy about the roll axis improved to with $8^{\circ}$ of the nadir. This time response clearly demonstrates the significant benefits of considering the inertia distribution when deriving the control law. Simply applying the torque-projection control without considering the inertia distribution is wholly unacceptable.

Figure 5 now illustrates the performance achievable when the weighted PD controller is augmented with the weighted feed-forward compensator. Pointing accuracy about the roll axis is improved by a further $3^{\circ}$ allowing regulation within $5^{\circ}$ of the nadir pointing attitude, well within the required specification. Figure 6 also shows the magnetic dipole time history, with the control inputs remaining comfortably within the saturation limits. 
Extensive simulation studies on the GOCE simulator has been carried on different initial conditions, different disturbance profiles and the variations of inertia up to $10 \%$ []. It shows consistent performance has been achieved for the proposed scheme with good stability and robustness.

\section{CONCLUSIONS}

This paper has presented a systematic approach to the design of a magnetic attitude control system for a satellite with an uneven inertia distribution. The traditional torqueprojection PD control approach has been investigated and shown to be inadequate for the regulation of satellites with an uneven inertia distribution. To tackle the weaknesses in the torque-projection approach, the problem of deriving the control torque is considered as a simple optimisation problem rather than a geometric one. The control torque is derived by solving a simple quadratic programming problem for which a closed-form solution is shown to exist.

The basic philosophy of the weighted feedback controller is then extended to derive a feed-forward controller to improve the disturbance rejection characteristics of the control law. A simple Kalman filter provides estimates of the external disturbance, with the feedforward controller then designed to minimise the overall accelerations due to these disturbances. 
Simulations on the high fidelity GOCE simulator demonstrate the effectiveness of the proposed controller. The attitude of the GOCE satellite is regulated within $5^{\circ}$ of the nadir when subject to significant disturbance torques due to the external environment. Furthermore this is carried out using a controller that is easy to implement. The proposed approach is expected to have a direct impact on industrial applications of magnetic attitude control systems of satellites with an uneven inertia distribution.

\section{ACKNOWLEDGEMENTS}

The authors would like to thank Drs Denis Fertin and Christian Philippe in the European space Agency (ESA) for their useful comments and provision of the GOCE simulator. They would also like to thank the ESA for financial support for this research.

\section{REFERENCES}

1 Martel, F., Pal, P., and Psiaki, M. Active Magnetic Control System for Gravity Gradient Stabilized Spacecraft. In 2nd Annular AIAA/USU Conference on Small Satellites, Logan, Utah, USA, 1988.

2 Arduini, C., and Baiocco, P. Active Magnetic Damping Attitude Control for Gravity Gradient Stabilized Spacecraft. Journal of Guidance and Control, 1997, 20(1), 117-122.

3 Wisniewski, R. Satellite Attitude Control Using Only Electromagnetic Actuation. Ph.D. Thesis, Aalborg University, Denmark, 1996. 
4 Lovera, M., De Marchi E., and Bittanti, S. Periodic Attitude Control Techniques for Small Satellite With Magnetic Actuators. IEEE Transactions on Control Systems Technology, 2002, 10(1), 90-95.

5 Psiaki, M. Magnetic Torquer Attitude Control via Asymptotic Periodic Linear Quadratic Regulation. AIAA Guidance, Navigation, and Control Conference, Denver, Colorado, USA, 2000.

6 Silani, E., and Lovera, M. Magnetic Spacecraft Attitude Control: A Survey and Some New Results. Control Engineering Practice, 2005, 13, 357-371.

7 Wood, M. and Chen W.H. Attitude Regulation of Magnetically Actuated Satellites using Model Predictive Control with Disturbance Modelling. Proceedings of IEEE Conference on Networking, Sensing and Control, China, 2008.

8 Wood, M. and Chen W.H. Model Predictive Control of Low Earth Orbiting Satellites Using Magnetic Actuation. Proceedings of the Institute of Mechanical Engineers, Part I: Journal of Systems and Control Engineering, in press, 2008.

9 Sechi, G., André, G., Andreis, D., and Saponara, M. Magnetic Attitude Control of the GOCE Satellite. Proceedings of the 6th International ESA Conference on Guidance, Navigation and Control Systems, Loutraki, Greece, 2006. 
10 Luenberger, D. Linear and Non-Linear Programming. Addison-Wesley, Reading, MA.

11 Pittelkau, M. Optimal periodic control for spacecraft pointing and attitude determination. Journal of Guidance, Control and Dynamics, 1993, 16(6), 10781084.

12 Wood, M. Attitude control of magnetically actuated satellites using optimisation. Ph.D. thesis, Department of Aeronautical and Automotive Engineering, Loughborough University, UK. 2009.

Table 1 - Satellite inertia distribution

\begin{tabular}{cc}
\hline Satellite Principal Axis & Inertia kgm $^{2}$ \\
\hline Roll $\left(\mathrm{I}_{\mathrm{x}}\right)$ & 152.2 \\
\hline Pitch $\left(\mathrm{I}_{\mathrm{y}}\right)$ & 2690.8 \\
\hline Yaw $\left(\mathrm{I}_{\mathrm{z}}\right)$ & 2652.6 \\
\hline
\end{tabular}

Table 2 - Torque projection PD controller

\begin{tabular}{l|c|c}
\hline & $\begin{array}{c}\text { Commanded Torque } \\
(\mu \mathrm{Nm})\end{array}$ & $\begin{array}{c}\text { Implemented Torque } \\
(\mu \mathrm{Nm})\end{array}$ \\
\hline Roll axis & -1.64 & 8.16 \\
\hline Pitch axis & -36.83 & -36.83 \\
\hline Yaw axis & 36.30 & 2.36 \\
\hline
\end{tabular}


Table 3 - Comparison of torque projection and weighted PD controller

\begin{tabular}{l|c|c|c}
\hline & $\begin{array}{c}\text { Commanded Torque } \\
(\mu \mathrm{Nm})\end{array}$ & $\begin{array}{c}\text { Torque Projection } \\
(\mu \mathrm{Nm})\end{array}$ & $\begin{array}{c}\text { Weighted PD } \\
(\boldsymbol{\mu N \boldsymbol { N }})\end{array}$ \\
\hline Roll axis & -1.64 & 8.16 & $\mathbf{- 0 . 3 3}$ \\
\hline Pitch axis & -36.83 & -36.83 & $\mathbf{- 3 6 . 8 3}$ \\
\hline Yaw axis & 36.30 & 2.36 & $\mathbf{- 0 . 0 9}$ \\
\hline
\end{tabular}

Table 4 - Effect of feed-forward compensation

\begin{tabular}{l|c|c|c}
\hline & \multicolumn{3}{|c}{ Disturbance torque after feed-forward compensation $(\mathrm{Nm})$} \\
\hline Strategy & Uncompensated & Torque Projection & Weighted \\
\hline Roll axis & $1 \mathrm{e}-6$ & $-2.6 \mathrm{e}-6$ & $\mathbf{- 1 e - 8}$ \\
\hline Pitch axis & $1 \mathrm{e}-5$ & 0 & $\mathbf{0}$ \\
\hline Yaw axis & $1 \mathrm{e}-5$ & $9 \mathrm{e}-6$ & $\mathbf{9 . 7 e - 6}$ \\
\hline
\end{tabular}
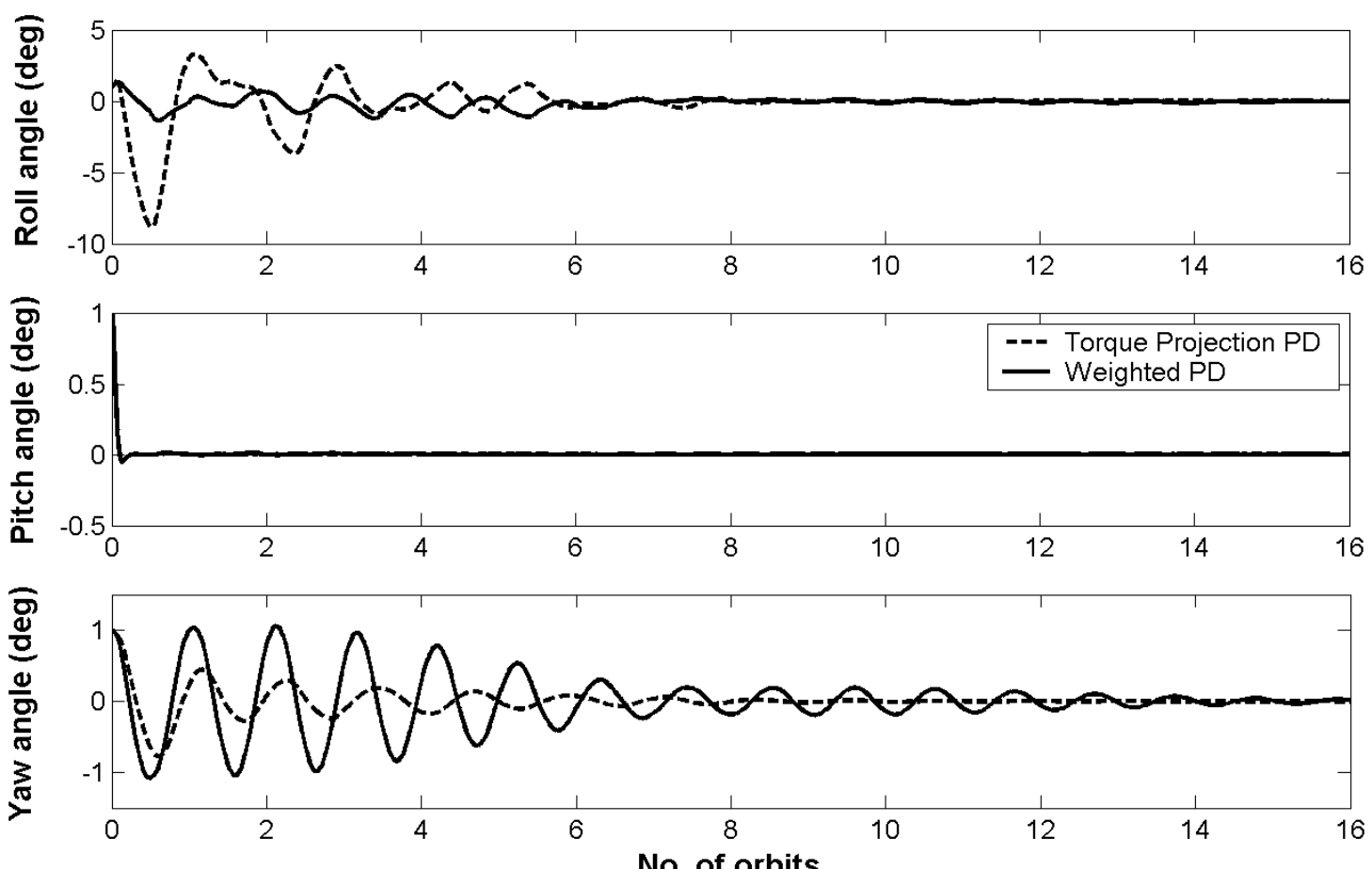

Fig. 1 - Response under torque projection and weighted PD controllers 

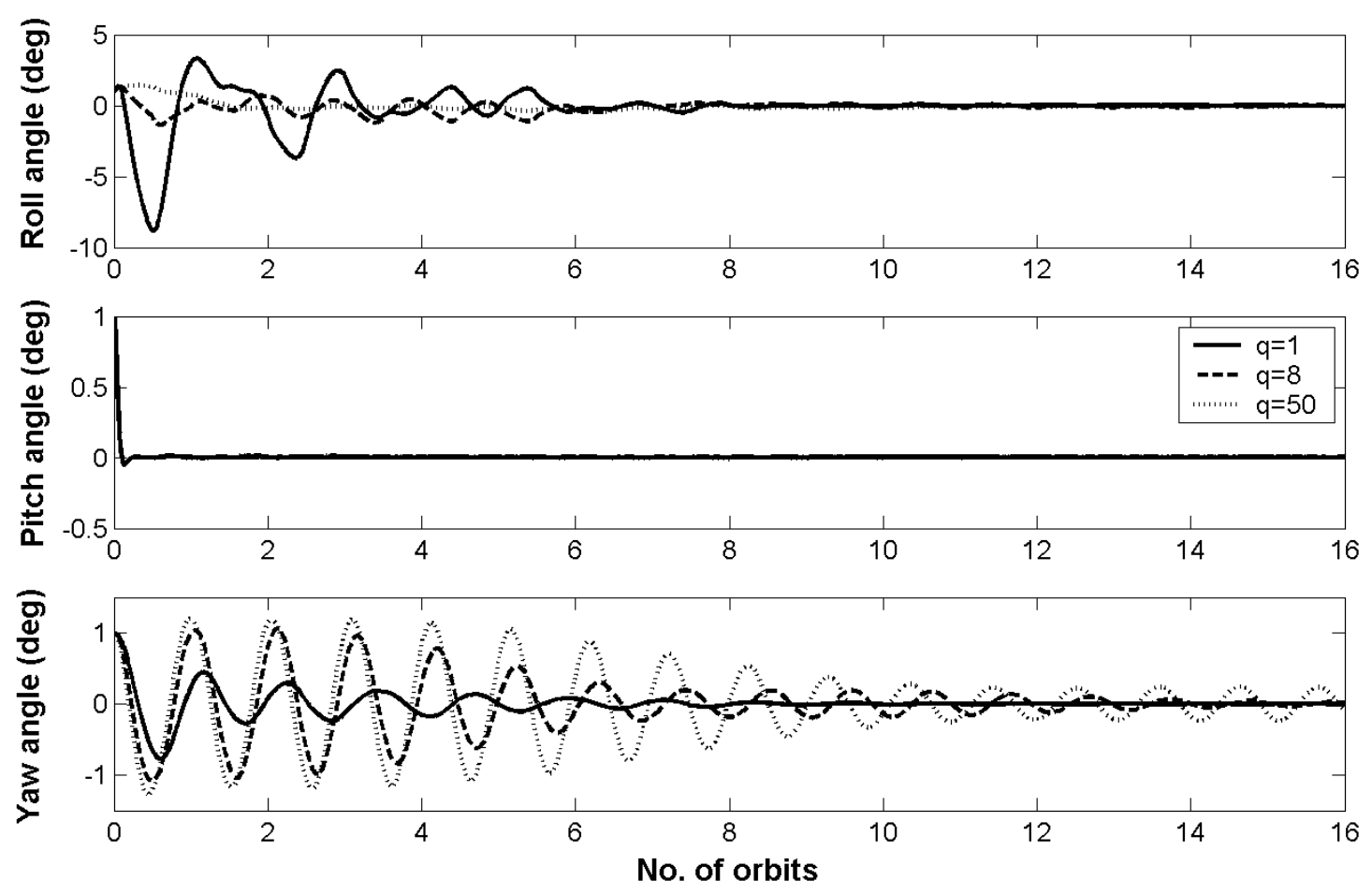

Fig. 2 - Effect of varying roll weighting factor
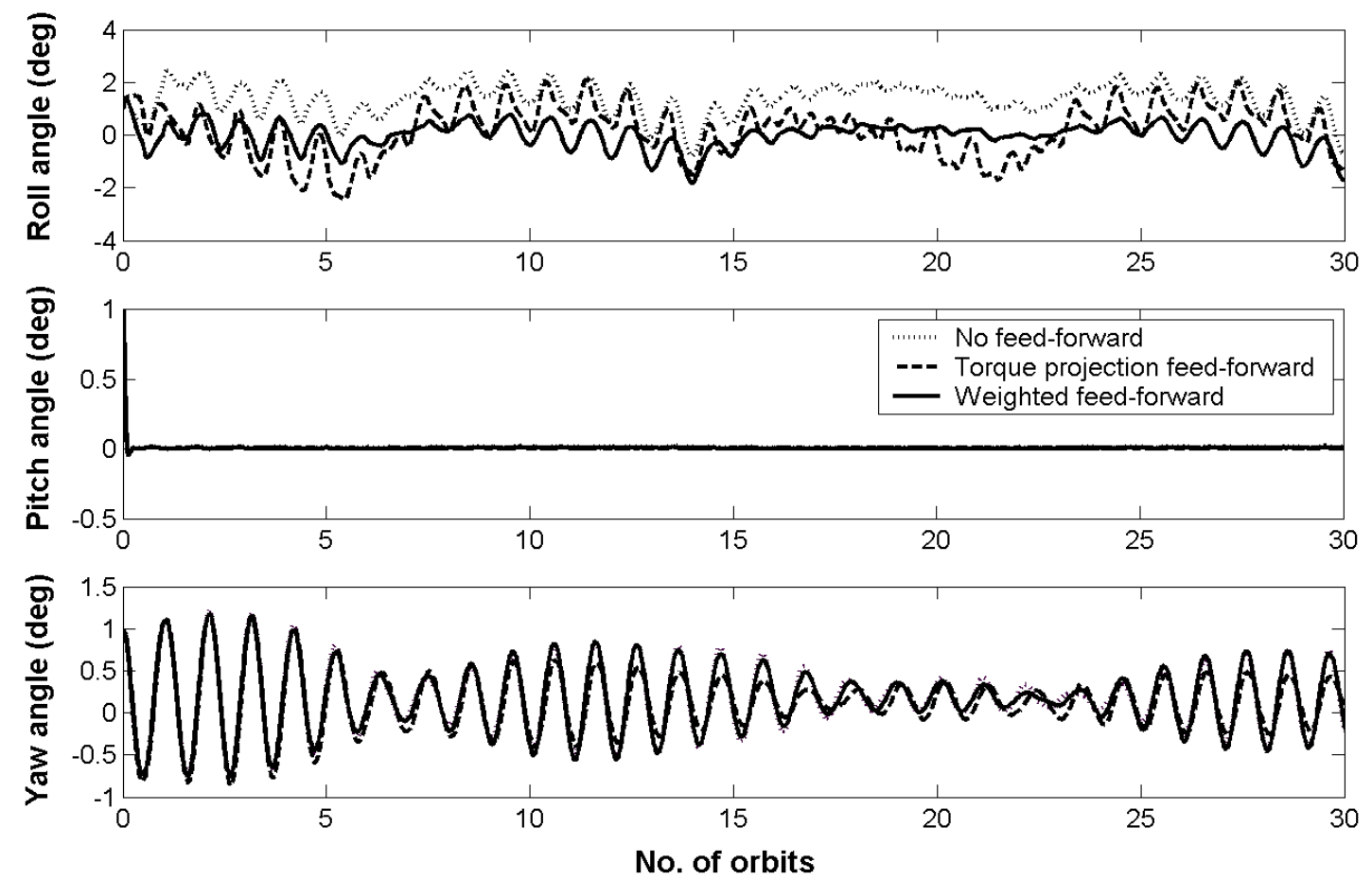

Fig. 3 - Comparison of feed-forward strategies 

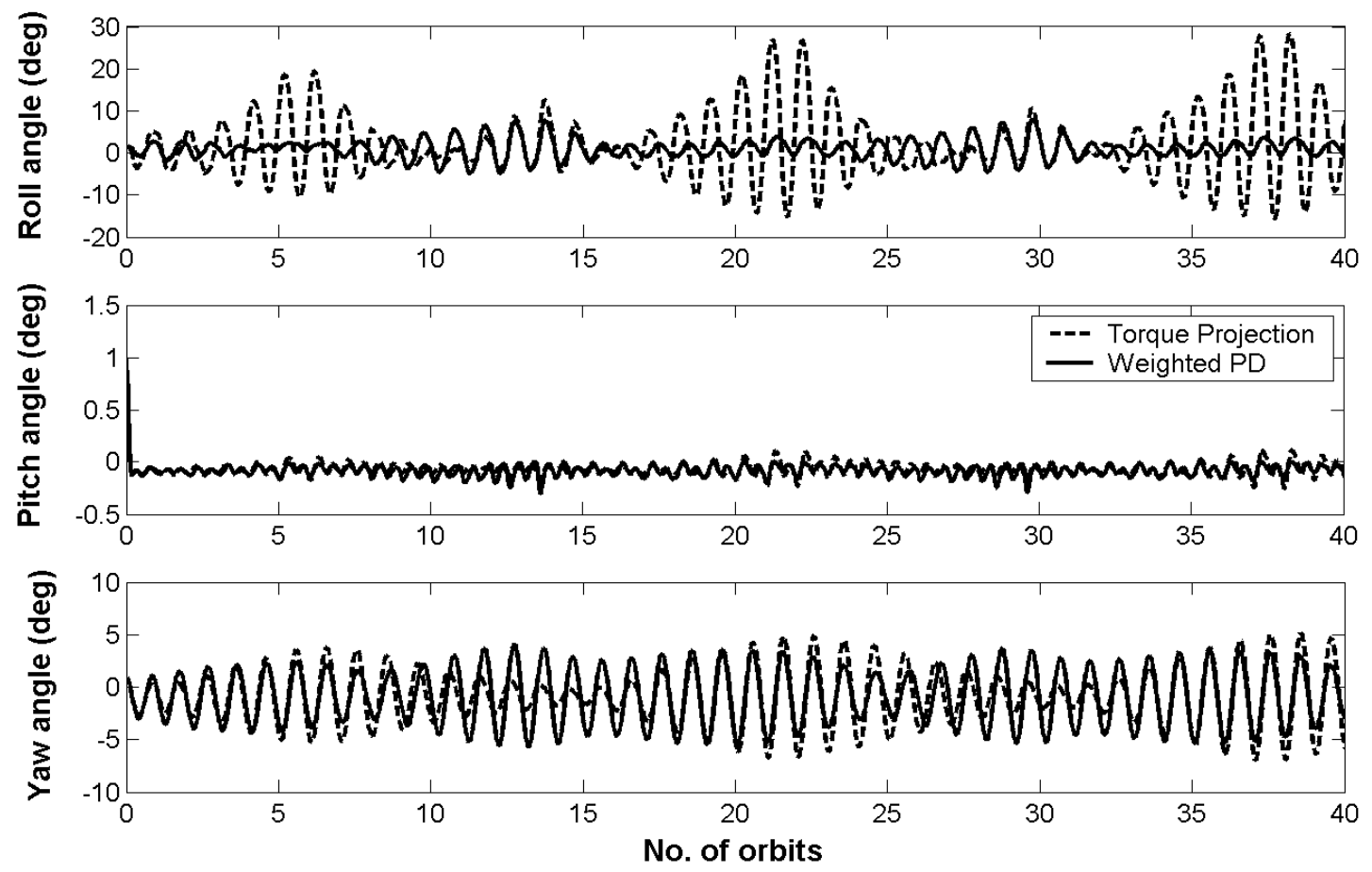

Fig.4 - Comparison of weighted and torque projection PD with external disturbance
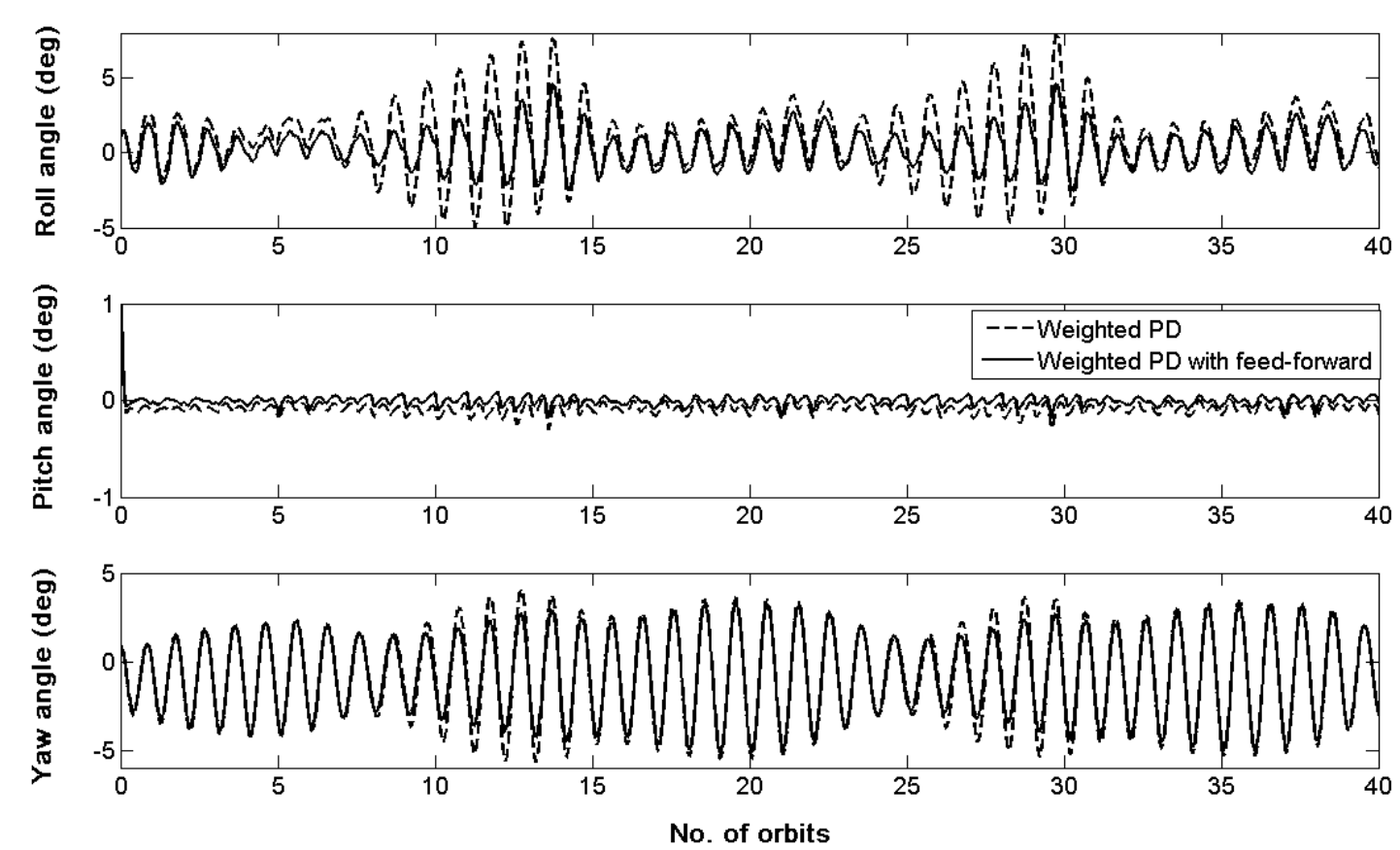

Fig. 5 - Performance under weighted PD with weighted feed-forward control 

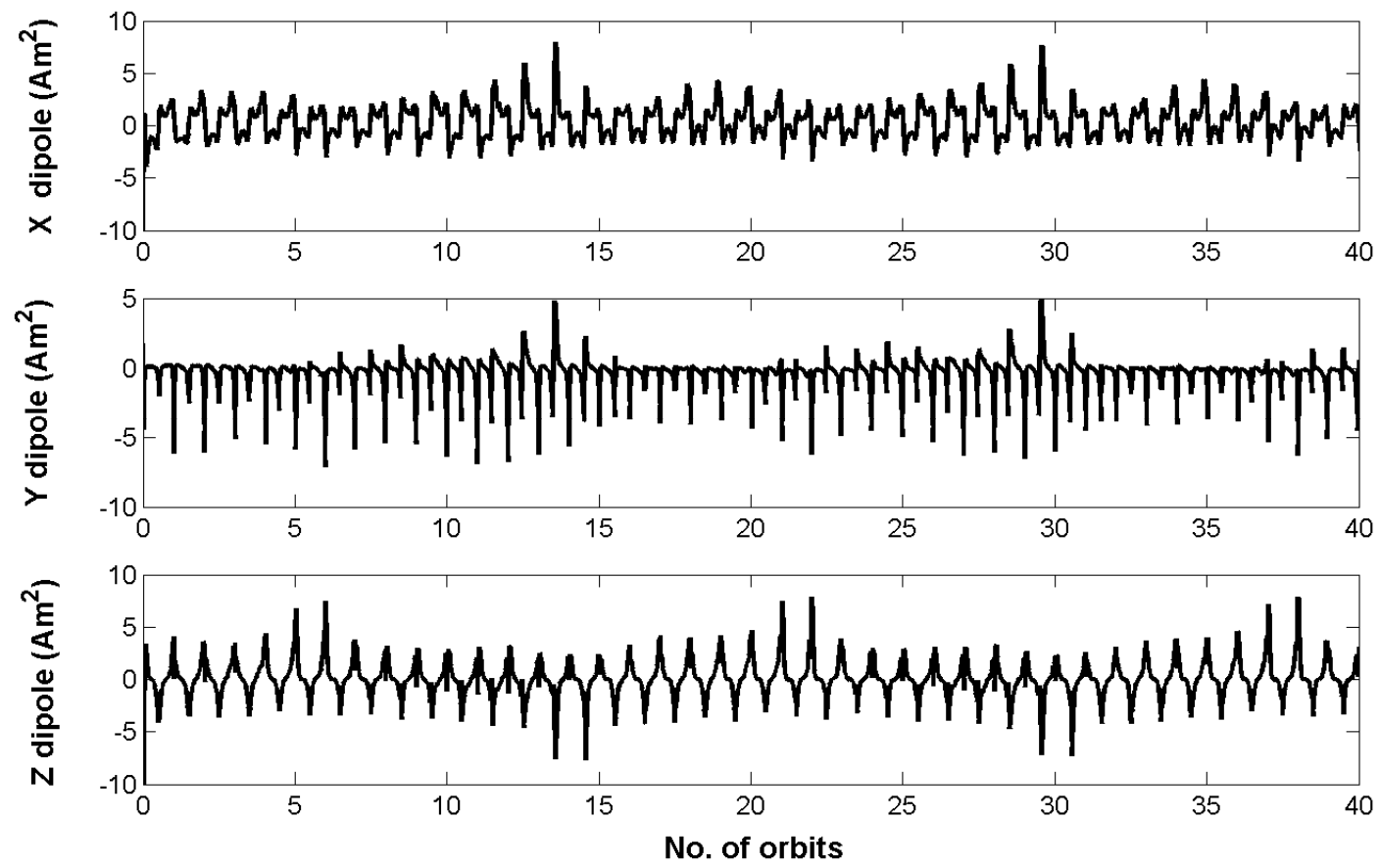

Fig. 6 - Magnetic dipole history

\section{APPENDIX}

\section{Notation}

A

Discrete-time system matrix for satellite attitude dynamics

A $\quad$ Continuous-time system matrix for satellite attitude dynamics

A $_{\mathbf{e}} \quad$ Discrete-time system matrix of augmented plant model

$a$

Orbit height

B Discrete-time control matrix for satellite attitude dynamics

B $\quad$ Continuous-time control matrix for satellite attitude dynamics

B $_{\mathrm{e}} \quad$ Discrete-time control matrix of augmented plant model

$\boldsymbol{B}_{m a g} \quad$ Earth's magnetic field vector in spacecraft coordinates 


\begin{tabular}{|c|c|}
\hline$i_{m}$ & Orbital inclination \\
\hline $\mathbf{J}$ & Satellite inertia matrix \\
\hline $\mathbf{K}$ & Ideal feedback gain matrix \\
\hline $\mathbf{L}_{\mathbf{e}}$ & Kalman filter gain matrix \\
\hline$M$ & Magnetic dipole vector \\
\hline $\mathbf{Q}$ & Weighting matrix for determination of optimal feedback control \\
\hline $\mathbf{Q}_{\mathrm{FF}}$ & Weighting matrix for determination of optimal feed-forward control \\
\hline$T_{\text {ideal }}, T_{\text {true }}$ & Ideal and true feedback control torque \\
\hline$T_{F F_{\text {ideal }}}, T_{F F_{\text {true }}}$ & Ideal and true feed-forward control torque \\
\hline $\boldsymbol{x}$ & State vector \\
\hline$x_{e}$ & Augmented state vector \\
\hline$\sigma_{1}, \sigma_{2}, \sigma_{3}$ & Inertia ratios \\
\hline$\varphi, \theta, \psi$ & Pointing angle of spacecraft about local-level coordinate frame \\
\hline$\omega_{x}, \omega_{y}, \omega_{z}$ & Angular rate of spacecraft about local-level coordinate frame \\
\hline$\omega_{0}$ & Orbital rate \\
\hline$\mu_{f}$ & Magnetic field dipole strength \\
\hline$\lambda$ & Lagrange multiplier \\
\hline
\end{tabular}

